\title{
Emotional distraction unbalances visual processing
}

\author{
Rashmi Gupta $\cdot$ Jane E. Raymond
}

Published online: 7 January 2012

(C) Psychonomic Society, Inc. 2012

\begin{abstract}
Brain mechanisms used to control nonemotional aspects of cognition may be distinct from those regulating responses to emotional stimuli, with activity of the latter being detrimental to the former. Previous studies have shown that suppression of irrelevant emotional stimuli produces a largely right-lateralized pattern of frontal brain activation, thus predicting that emotional stimuli may invoke temporary, lateralized costs to performance on nonemotional cognitive tasks. To test this, we briefly $(85 \mathrm{~ms})$ presented a central, irrelevant, expressive (angry, happy, sad, or fearful) or neutral face $100 \mathrm{~ms}$ prior to a letter search task. The presentation of emotional versus neutral faces slowed subsequent search for targets appearing in the left, but not the right, hemifield, supporting the notion of a right-lateralized, emotional response mechanism that competes for control with nonemotional cognitive processes. Presentation of neutral, scrambled, or inverted neutral faces produced no such laterality effects on visual search response times.
\end{abstract}

Keywords Attention - Emotion · Faces · Laterality

Irrelevant emotional or arousing stimuli (e.g., a billboard advertising a new thriller) frequently demand ignoring as more mundane, less emotional tasks are pursued. Suppressing responses to these stimuli requires cognitive control, or regulation of effort, because, as numerous studies have shown, emotional stimuli are highly effective at drawing attention away from task-relevant information, thereby interfering with performance on other ongoing tasks (e.g.,

\section{R. Gupta $\cdot$ J. E. Raymond $(\bowtie)$}

School of Psychology, Bangor University,

Brigantia Building, Penrallt Road,

Bangor, Gwynedd LL57 2AS, UK

e-mail: j.raymond@bangor.ac.uk
Aquino \& Arnell, 2007; Most, Chun, Widders, \& Zald, 2005; Srinivasan \& Gupta, 2010; Whalen et al., 1998). Typically, studies demonstrating emotional interference effects measure performance on simple nonemotional tasks, such as word color naming (e.g., Williams, Mathews, \& MacLeod, 1996) or word counting (Bush, Luu, \& Posner, 2000; Whalen et al., 1998), locating simple stimuli (as in dot-probe tasks; e.g., Lipp \& Derakshan, 2005), or performing simple mental operations (e.g., Schimmack, 2005). Performance decrements found when emotional (but not nonemotional), irrelevant stimuli are concurrently present are thought to reflect interference of emotionally responsive brain mechanisms on those required to perform nonemotional tasks.

A prevailing explanation for these emotional interference effects is that emotional stimuli alter the top-down processing biases used to determine access of sensory information to limited-capacity processing resources that enable overt responses (Öhman, Lundqvist, \& Esteves, 2001). In this view, emotional stimuli act like highly salient stimuli that draw central resources away from the nonemotional task, leading to performance decrements. Some neuroimaging and clinical studies have proposed that the mechanisms controlling processing of emotional stimuli are neurologically distinct from those controlling nonemotional cognition and that activation of the former causes suppression of the latter (Bush et al., 2000; Drevets \& Raichle, 1998; Yamasaki, LaBar, \& McCarthy, 2002). An extreme view of this seesaw relationship between mechanisms controlling emotional and nonemotional processing is the freezing account of emotional interference, which posits that emotional stimuli cause a temporary freezing of all ongoing activity (Flykt, 2006; Öhman, Flykt, \& Esteves, 2001).

Supporting a dichotomous view of emotional versus nonemotional cognition, Dolcos and McCarthy (2006) reported 
selective activation of a ventral prefrontal cortex emotional cognition system when irrelevant emotional (but not neutral) images were presented during the retention interval of a simple visual working memory task. Interestingly, they found, like numerous other studies (Adolphs, Damasio, Tranel, \& Damasio, 1996; Borod et al., 1998; Keil et al., 2003), that activation was significantly greater in the right versus left hemisphere. (Note: In contrast, FusarPoli et al., 2009, conducted a meta-analysis of 105 fMRI studies involving emotional faces and found no evidence for a right-hemisphere bias in activation. However, they included studies regardless of attentional allocation to faces, face relevance to goals, or the duration of face presentation, limiting applicability to the present issue.) If a cognitive system specifically sensitive to emotional content were right lateralized and it were to compete in a seesaw manner with cognitive mechanisms normally used for nonemotional tasks (Bush et al., 2000; Drevets \& Raichle, 1998), specific predictions that have not been previous investigated could be made with regard to performance on speeded visual cognition tasks. Namely, performance on a lateralized nonemotional letter search task should be worse for left visual field (LVF) versus right visual field (RVF) targets if the search array were preceded by a central emotional distracting image (prime), with no such imbalance occurring if the prime were nonemotional. A prime with emotional content should induce greater right- than left-hemisphere activity, which, in turn, should reduce performance for LVF versus RVF targets. However, if affectively sensitive processes were not lateralized but still had a seesaw relationship with processes responsive to nonemotional stimuli, performance should be impaired with emotional, but not neutral, primes, regardless of target hemifield. If emotional processes do not compete with processes responding to nonemotional stimuli, there should be no difference in performance when distractors are emotional or neutral, especially if they are generally matched on complexity, contrast, and other stimulus features. The aim of our experiment was to test these possibilities. Two somewhat relevant previous studies reported an LVF performance decrement after or during exposure to emotional stimuli (Hartikainen, Ogawa, \& Knight, 2000; Thompson, Malloy, Cone, \& Hendrickson, 2009), but in both cases, such effects could have been due to eye movement biases, spatial attention shifts, or featural differences between emotional and nonemotional stimuli.

To overcome these shortfalls and to more directly test for the presence of a lateralized, rapidly acting seesaw mechanism, we presented a large emotionally expressive or neutral face $100 \mathrm{~ms}$ prior to the presentation of a simple letter search task that was specifically designed to probe lateralization of visual processing. Six letters were presented, three to the left and three to the right of fixation (Fig. 1); the participant's task was to report whether an $\mathrm{X}$ or an $\mathrm{N}$ was present on each trial. We used faces as distracting primes, rather than the International Affective Picture System images used in previous studies (Dolcos \& McCarthy, 2006; Hartikainen et al., 2000), because facial expressions do not introduce substantive differences in low-level stimulus features and, therefore, are more appropriate for isolating emotional versus nonemotional responses. We introduced a brief temporal separation between the irrelevant face prime and the letter array to provide enough time for expression-specific activation to occur (see Eimer \& Holmes, 2007, for a review) but insufficient time for an eye movement. Unlike Hartikainin et al., we presented central rather than lateralized distractors to ensure that both hemispheres were equally stimulated. Distractors had one of five different emotional expressions (neutral, happy, angry, sad, and fearful) to explore the role of emotional valence (positive, negative), as well as arousal (fearful, sad). Different theories of emotion make different predictions in this regard. Some predict greater right-hemisphere activation regardless of valence or arousal (Adolphs et al., 1996; Borod et al., 1998); others posit that positive and negative emotions should preferentially engage the left and right hemispheres, respectively (Davidson, 1992; Gur, Skolnick, \& Gur, 1994); still others suggest that the nature of response (approach, avoid) should determine emotional laterality (Davidson \& Irwin, 1999). A second, control experiment, using upright and inverted neutral faces, a scrambled face, and a simple oval as primes, assessed laterality effects with nonemotional primes.

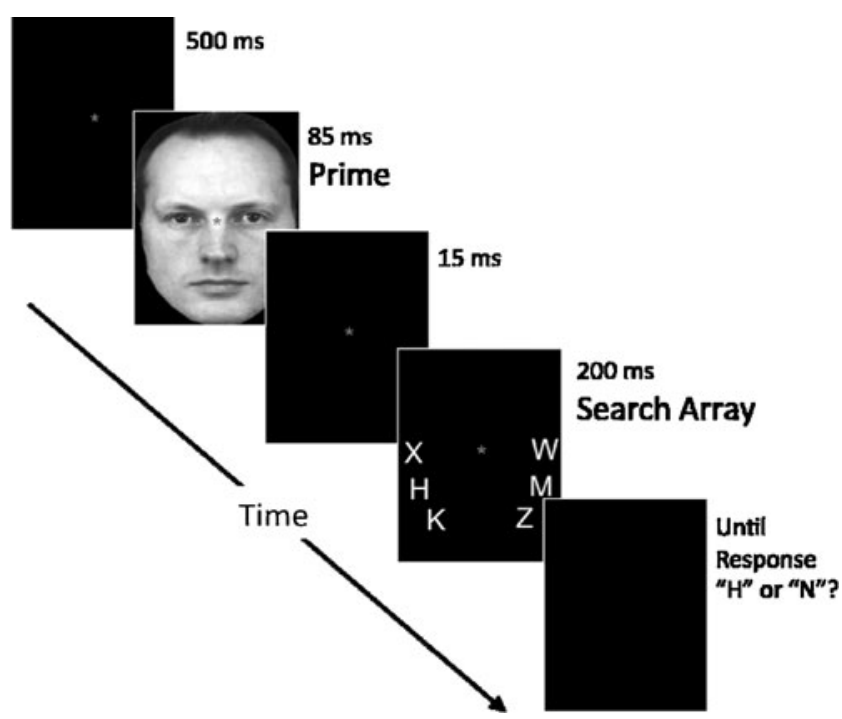

Fig. 1 An example trial. The prime face was displayed for $85 \mathrm{~ms}$, followed by a 15 -ms blank. The letter search array appeared, configured as shown in the figure. The task was to report as soon as possible whether the target was an $\mathrm{X}$ or an $\mathrm{N}$. The prime face had a neutral, happy, angry, sad, or fearful expression 


\section{General method}

Participants

In exchange for course credit, printer credits, or money, 40 right-handed students (Experiment 1, $N=21,7$ males, mean age $=24.7$ years; Experiment $2, N=19,11$ males, mean age $=$ 23.1 years) reporting normal or corrected-to-normal vision participated after giving informed consent.

\section{Apparatus}

Stimuli were displayed on a 23 -in. color monitor $(85 \mathrm{~Hz}, 32-$ bit true color; resolution, 1,289 × 1,024 pixels) viewed binocularly from a distance of $70 \mathrm{~cm}$. E-Prime software (Version 1.1) operating on a computer with a $3.4-\mathrm{GHz}$ Pentium 4 processor generated stimuli and recorded responses acquired via a number pad.

\section{Stimuli}

Six upright grayscale faces $\left(8.5^{\circ} \times 5.3^{\circ}\right)$ of young adults (three males, three females) bearing a happy, angry, sad, fearful, or neutral expression, with neither teeth nor neck visible, served as primes in Experiment 1. The same set (neutral expressions only) served as primes in Experiment 2 and were presented upright or inverted. Additionally, an open oval $\left(8.5^{\circ}\right.$ tall, $5.7^{\circ}$ wide, line width $0.1^{\circ}$ ) and a scrambled face (created by segmenting a single face into a $4 \times 5$ grid and then arranging the segments randomly) also served as primes. The visual search array comprised six letters simultaneously displayed in light green (Arial font; $0.65^{\circ} \times$ $0.57^{\circ}$ ) on a black field arranged as shown in Fig. 1. Each letter was separated from its nearest neighbor by $1.6^{\circ}$. Targets were either $\mathrm{X}$ or $\mathrm{N}$; distractors were $\mathrm{H}, \mathrm{K}$, $\mathrm{M}, \mathrm{W}$, or $\mathrm{Z}$.

\section{Experimental design and procedure}

Each trial began with the presentation of a 500-ms central fixation point, followed by an 85-ms prime, a 15-ms blank screen, and then a 200-ms letter array (see Fig. 1). The brevity of this display was used to prevent eye movements from being a useful strategy. Participants were asked to maintain central fixation, to ignore the irrelevant primes, and to identify the target letter ( $\mathrm{X}$ or $\mathrm{N}$ ) as quickly as possible. " $\mathrm{X}$ " and " $\mathrm{N}$ " responses were indicated by depressing the " 2 " or " 8 " keys, respectively, on the number pad (arranged vertically to reduce spatial response compatibility issues), using the thumb and index finger, respectively, of the right hand. Within each experiment, each prime type, target hemifield (left or right), and target identity were equiprobable, with each combination occurring 18 times in a pseudorandomized order. Target location within each hemifield (upper, mid, lower) was also equiprobable. Six blocks of 60 or 48 trials each (Experiments 1 and 2, respectively) were completed, with average accuracy being provided to the participant at the end of each block. A full practice block, with feedback given on each trial, preceded the experimental session.

\section{Data analysis}

Data from 2 participants with excessively high error rates $(>25 \%)$ were excluded from each experiment. Data were excluded if response times (RTs) were less than $200 \mathrm{~ms}$ or more than $2,000 \mathrm{~ms}$ or targets were incorrectly identified (12\% of the data). Repeated measures analyses of variance (ANOVA) were conducted on individual condition mean RTs and error rates, using target hemifield (left, right), target letter (X-thumb, N-finger), and prime type as withinsubjects factors. Post hoc comparisons were made using planned $t$-tests. Alpha levels were set to .05 .

\section{Experiment 1}

Results

The aim was to investigate whether the presence of emotional content in the face prime would interact with target location (LVF vs. RVF) to determine performance on the letter search task. Indeed, as can be seen in Fig. 2, a significant interaction effect of prime type and target VF, $F(4,72)=3.372, p<.02$,

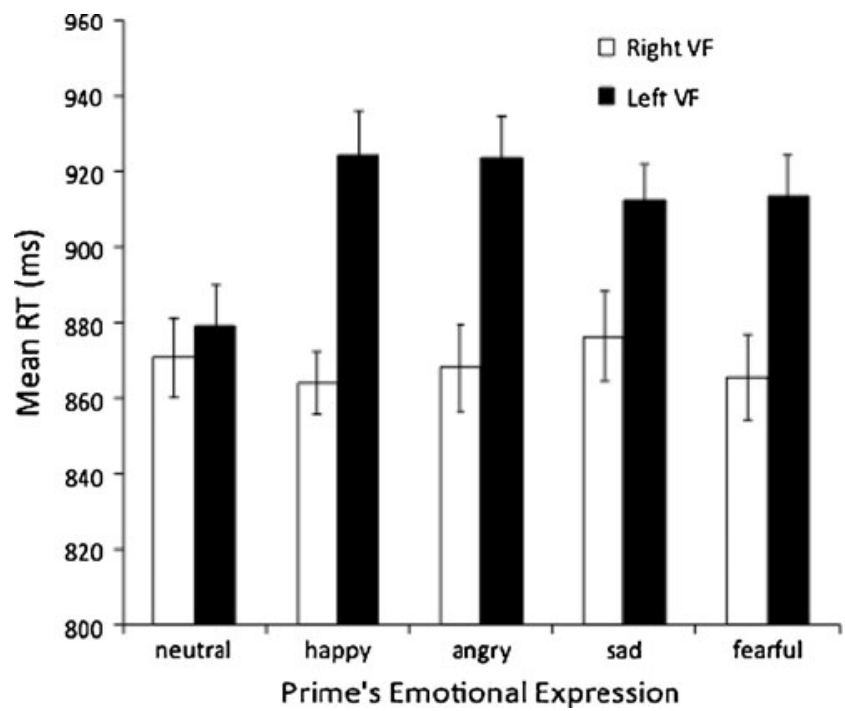

Fig. 2 Group mean response time (RT) obtained in Experiment 1 when letter targets were presented on the right (open bars) or left (black bars) for each prime facial expression. VF, visual field. Vertical lines represent \pm 1 within-subjects standard error 
$\eta_{\mathrm{p}}{ }^{2}=.158$, on mean RT was found. RTs for LVF targets were longer when the search array was preceded by an emotionally expressive face prime than when it was preceded by a neutral prime; RTs for RVF targets were unaffected by prime type. Although, on average, RTs were $41 \mathrm{~ms}$ shorter to RVF than to LVF targets, $F(1,18)=4.336, p=.052, \eta_{\mathrm{p}}{ }^{2}=.194$, and were $110 \mathrm{~ms}$ shorter when responses were made using the thumb versus index finger, $F(1,18)=32.474, p=.001, \eta_{\mathrm{p}}{ }^{2}=.643$, $\mathrm{VF}$ and response digit did not interact significantly, $F<1$, and more relevantly, the triple interaction (prime type, VF, and response digit) was also nonsignificant, $F(4,72)=1.684$, $p=.163, \eta_{\mathrm{p}}{ }^{2}=.086$.

Separate ANOVAs conducted on the mean RT obtained when the target was in the LVF or RVF, using prime type as a within-subjects factor, showed a nonsignificant main effect of prime type for RVF targets, $F<1$, but a significant effect for LVF targets, $F\left(4,72=4.925, p<.001, \eta_{\mathrm{p}}{ }^{2}=.215\right.$. Planned comparisons of mean RTs obtained in the expressive versus neutral prime conditions with LVF targets revealed significantly slower responses (relative to neutral) in all the expressive prime conditions (slowing effect: happy, $46 \mathrm{~ms}, p=.01$; angry, $45 \mathrm{~ms}, p=.01$; sad, $33 \mathrm{~ms}, p=.03$; fearful, $33 \mathrm{~ms}, p=.03$ ). For each participant and visual field, we quantified the emotional slowing effect as the difference between the average RT with emotional primes (all expressions) and the RT with neutral primes. Twelve of the 19 participants $(63 \%)$ showed greater emotional slowing for LVF versus RVF targets.

Error rates were modestly but significantly $(p<.05)$ higher for LVF $(12.5 \%)$ than for RVF $(9.6 \%)$, indicating that visual field effects on RT were not due to speedaccuracy trade-offs. Error rates were also significantly higher $(p<.05)$ for responses made with the thumb $(13.0 \%)$ versus finger $(9.1 \%)$, indicating that RT effects associated with response digit probably reflect such a trade-off. Importantly, the ANOVA of error rates showed that the main effect of prime type and all its interactions were nonsignificant (all $p \mathrm{~s}>.30$ ).

Although the search array was presented for only $200 \mathrm{~ms}$, we explored whether eye movements may have played a role in this experiment. Using the same stimuli and procedure, three right-handed participants performed the task while eye movements were recorded (using an Eyelink 1000 eye monitor). Saccades occurred rarely-on only $10 \%$ of the trials, on average. The mean latency of the first saccade after prime onset was 1,074 ms, approximately $700 \mathrm{~ms}$ after the letter array had disappeared and $200 \mathrm{~ms}$ before the manual response was executed. When they occurred, saccades were more likely to be left-directed (9\%) than right-directed $(1 \%)$. There was a modest tendency to make more saccades on trials with LVF versus RVF targets. For LVF targets, the likelihood of a left-directed saccade was similar for all prime types.

\section{Experiment 2}

An important question for studies aiming to study emotional interference effects is whether performance costs result from the emotional content of the irrelevant stimuli or, instead, simply reflect interference effects of stimuli with enhanced information value or sensory salience. If nonemotional content in distractors can account for performance modulations, there is no need to hypothesize separate mechanisms for emotional versus nonemotional cognition, with the activity of one interfering with the other. Experiment 2 explored this possibility by using four different nonemotional prime types that varied in complexity and ease of perceptual interpretation (upright neutral faces, inverted neutral faces, a scrambled face, and an open oval). The procedure was as for Experiment 1 with minor adjustments as described in the General Method section.

\section{Results}

Mean RTs obtained when targets appeared in the LVF and RVF in each prime condition are shown in Fig. 3. Target visual field had a nonsignificant effect on RT, $F<1$, and did not interact with prime type, $F(3,54)=1.312, p>.25, \eta_{\mathrm{p}}{ }^{2}=.068$. Even though primes differed in perceptual complexity and information load, there was no evidence of a visual field effect in any condition. Of less interest here, prime type had a significant main effect, $F(3,54)=11.195, p<.001, \eta_{\mathrm{p}}{ }^{2}=.383$, with the two intact face conditions producing significantly shorter RTs ( $p$ s $<.05$ in all cases) than did the nonface conditions (oval, scrambled), perhaps reflecting an arousal or alerting effect. Overall, responses were

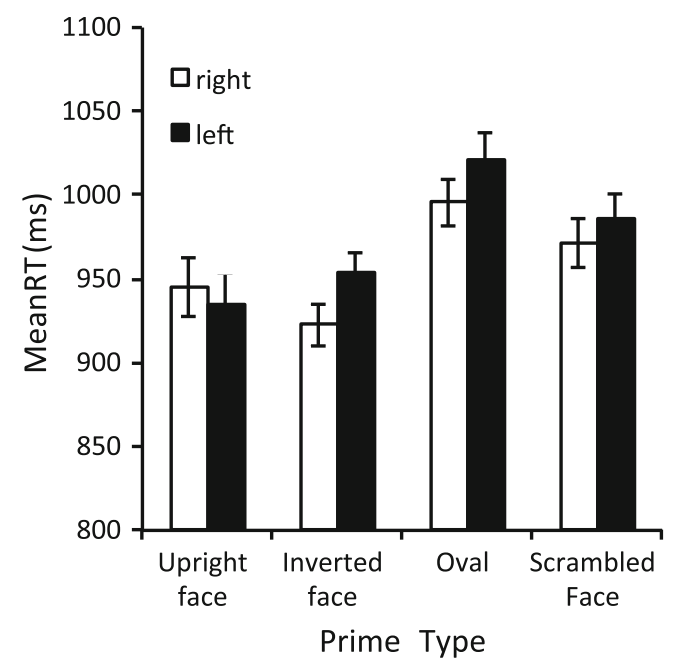

Fig. 3 Group mean response time (RT) obtained in Experiment 2 when letter targets were presented on the right (open bars) or left (black bars) for each type of prime. Vertical lines represent \pm 1 within-subjects standard error 
slower than in Experiment 1, perhaps because its primes were more arousing. More pertinent is that this follow-up experiment replicated the control condition in Experiment 1 by finding that when neutral face primes were used, mean RT did not depend on target visual field $(p>.25)$. This experiment provides no support for the notion that image complexity or ease of perceptual interpretation accounts for the lateralized performance differences seen with emotional face primes in Experiment 1.

\section{Discussion}

When an emotionally expressive versus neutral face prime was briefly presented just prior to the presentation of a bilateral visual search array, participants were slower at identifying a target if it appeared in the LVF. Identifying RVF targets was unaffected by the emotional content in the prime. The emotional slowing effect seen for LVF targets was similar for all the emotional expressions tested and was absent when nonemotional stimuli (including nonface stimuli) were presented. The results of Experiment 2 support the contention that there is an emotional basis to the lateralized interference effects found in Experiment 1 and that these effects were not due to stimulus complexity or information load. These simple experiments thus demonstrate a robust lateralized emotional interference effect and offer a straightforward technique for assessing emotional distraction.

Dichotomous models of cognitive control that posit competition between emotionally responsive cognitive mechanisms and nonemotional mechanisms offer a reasonable account for these effects. Previous neuroimaging studies reporting greater right than left activation to centrally presented, irrelevant emotional (vs. neutral) complex images during the retention interval of a working memory task (Dolcos \& McCarthy, 2006) support long-standing notions that cognitive mechanisms sensitive to emotional content are right-hemisphere biased (e.g., Ley \& Bryden, 1979). If the brief emotional primes we used stimulated the right more than the left hemisphere, they could have transiently disrupted visual processing mechanisms in the right hemisphere, slowing responses to LVF letter targets. In other words, engagement of the right hemisphere's emotionally responsive mechanism appears to have immediate, negative consequences for nonemotional cognitive processing of LVF information.

Two previous studies are consistent with this finding. Hartikainen et al. (2000) also reported slower responses to detect the appearance of a single triangle presented in the LVF versus RVF after the presentation of a single emotional image to the left or right of fixation. Unfortunately, their lateralized emotional primes presented $300 \mathrm{~ms}$ prior to the target both provided a spatial cue to attention, resulting in a complicated interaction of cue-target compatibility, prime, and target VF, and provided an opportunity for an eye movement. Moreover, they did not control for stimulus complexity in the primes, adding to the difficulty of interpreting their result. An LVF effect of emotional interference was also reported by Thompson et al. (2009), who measured speeded detection of a single dot appearing on a static face while participants listened to emotional versus nonemotional speech. They reported slowed responses to LVF, but not RVF, dot presentations when emotional, but not nonemotional, prosody was concurrent in the speech stream, suggesting that lateralized emotional interference effects may operate cross-modally.

Although it is tempting to posit that emotional stimuli induce a rapid lateral shift in attention toward the RVF (causing RT slowing for LVF targets), such a mechanism cannot explain our data, for two reasons. First, examination of eye movements in this task (even though rare and produced well after the display had disappeared) showed that overt orienting was more likely to be directed toward the left, not the right, predicting slowing for RVF, not LVF, targets. Spatial shifts in attention typically accompany or precede oculomotor shifts and have the effect of speeding responding (Awh, Armstrong, \& Moore, 2006). Second, if spatial attention had been shifted to one hemifield or the other by the presentation of an emotional prime, opposite effects on RT in the other hemifield should have been observed. The total absence of an RT effect of emotional primes for RVF targets argues against a spatial attention shift explanation for our findings.

Interestingly, we found no difference in the magnitude of the slowing effect depending on the nature of the emotional expression in the prime. Such a finding is consistent with the results of several previous studies of emotional interference effects that have reported similar interference effects regardless of valence (De Houwer \& Tibboel, 2010; Schimmack, 2005). Although a null effect of positive versus negative (happy, angry) and high versus low arousal (fear, sad) primes (all $p \mathrm{~s}>.30$ ) is difficult to interpret, our finding supports the view that emotional stimuli, if sufficiently salient, can engage a right-hemisphere emotional control system regardless of valence. Our results also do not support a lateralized approach-avoid account of emotional control (Davidson \& Irwin, 1999), because this notion posits that approach responses (as used in our study) should show performance benefits for RVF targets preceded by happy primes and costs with negative (especially fearful) primes, an effect we did not observe.

Acknowledgements This research is supported by a postdoctoral fellowship to R. Gupta from IBRO and by a grant from BBSRC (U.K.) to J. R. (BB/G021538/1). 


\section{References}

Adolphs, R., Damasio, H., Tranel, D., \& Damasio, A. R. (1996). Cortical systems for the recognition of emotion in facial expressions. Journal of Neuroscience, 16, 7678-7687.

Aquino, J. M., \& Arnell, K. M. (2007). Attention and the processing of emotional words: Dissociating effects of arousal. Psychonomic Bulletin \& Review, 14, 430-435.

Awh, E., Armstrong, K. M., \& Moore, T. (2006). Visual and oculomotor selection: Links, causes and implications for spatial attention. Trends in Cognitive Sciences, 10, 124-130.

Borod, J. C., Obler, L. K., Erhan, H. M., Grunwald, I. S., Cicero, B. A., Welkowitz, J., \& Whalen, J. R. (1998). Right hemisphere emotional perception: Evidence across multiple channels. Neuropsychology, 12, 446-458.

Bush, G., Luu, P., \& Posner, M. I. (2000). Cognitive and emotional influences in anterior cingulate cortex. Trends in Cognitive Sciences, 4, 215-222.

Davidson, R. J. (1992). Anterior cerebral asymmetry and the nature of emotion. Brain and Cognition, 20, 125-151.

Davidson, R. J., \& Irwin, W. (1999). The functional neuroanatomy of emotion and affective style. Trends in Cognitive Sciences, 3, 1121.

De Houwer, J., \& Tibboel, H. (2010). Stop what you are not doing! Emotional pictures interfere with the task not to respond. Psychonomic Bulletin \& Review, 17, 699-703.

Dolcos, F., \& McCarthy, G. (2006). Brain systems mediating cognitive interference by emotional distraction. Journal of Neuroscience, 26, 2072-2079.

Drevets, W. C., \& Raichle, M. E. (1998). Reciprocal suppression of regional cerebral blood flow during emotional versus higher cognitive processes: Implications for interactions between emotion and cognition. Cognition and Emotion, 12, 353-385.

Eimer, M., \& Holmes, A. (2007). Event-related brain potential correlates of emotional face processing. Neuropsychologia, 45, 15-31.

Flykt, A. (2006). Preparedness for action: Responding to the snake in the grass. American Journal of Psychology, 119, 29-43.

Fusar-Poli, P., Placentino, A., Carletti, F., Allen, P., Landi, P., Abbamonte, M., \& Politi, P. L. (2009). Laterality effect on emotional faces processing: ALE meta-analysis of evidence. Neuroscience Letters, 452, 262-267.

Gur, R. C., Skolnick, B. E., \& Gur, R. E. (1994). Effects of emotional discrimination tasks on cerebral blood flow: Regional activation and its relation to performance. Brain and Cognition, 25, 271286.

Hartikainen, K. M., Ogawa, K. H., \& Knight, R. T. (2000). Transient interference of right hemispheric function due to automatic emotional processing. Neuropsychologia, 38, 1576-1580.

Keil, A., Gruber, T., Muller, M. M., Moratti, S., Stolarova, M., Bradley, M. M., \& Lang, P. J. (2003). Early modulation of visual perception by emotional arousal: Evidence from steady-state visual evoked brain potentials. Cognitive, Affective, \& Behavioral Neuroscience, 3, 195-206.

Ley, R. G., \& Bryden, M. P. (1979). Hemispheric differences in processing emotions and faces. Brain and Language, 7, 127-138.

Lipp, O. V., \& Derakshan, N. (2005). Attentional bias to pictures of fear-relevant animals in a dot probe task. Emotion, 5, 365-369.

Most, S. B., Chun, M. M., Widders, D. M., \& Zald, D. H. (2005). Attentional rubbernecking: Cognitive control and personality in emotion induced blindness. Psychonomic Bulletin \& Review, 12, 654-661.

Öhman, A., Flykt, A., \& Esteves, F. (2001). Emotion drives attention: Detecting the snake in the grass. Journal of Experimental Psychology: General, 130, 466-478.

Öhman, A., Lundqvist, D., \& Esteves, F. (2001). The face in the crowd revisited: A threat advantage with schematic stimuli. Journal of Personality and Social Psychology, 80, 381-396.

Schimmack, U. (2005). Attentional interference effects of emotional pictures: Threat, negativity, or arousal? Emotion, 5, 55-66.

Srinivasan, N., \& Gupta, R. (2010). Emotion-attention interactions in recognition memory for distractor faces. Emotion, 10, 207-215.

Thompson, L. A., Malloy, D. M., Cone, J. M., \& Hendrickson, D. L. (2009). The face-to-face light detection paradigm: A new methodology for investigating visuospatial attention across different face regions in live face-to-face communication settings. Interaction Studies, 11, 336-348.

Whalen, P. J., Bush, G., McNally, R. J., Wilhelm, S., McInerney, S. C., Jenike, M. A., \& Rauch, S. L. (1998). The emotional counting Stroop paradigm: A functional magnetic resonance imaging probe of the anterior cingulate affective division. Biological Psychiatry, 44, 1219-1228.

Williams, J. M., Mathews, A., \& MacLeod, C. (1996). The emotional Stroop task and psychopathology. Psychological Bulletin, 120, 3 14

Yamasaki, H., LaBar, K. S., \& McCarthy, G. (2002). Dissociable prefrontal brain systems for attention and emotion. Proceeding of the National Academy of Sciences, 99, 11447-11451. 